\title{
REMARKS ON $t$-QUASICONVEX FUNCTIONS
}

\section{KAZIMIERZ NiKODEM AND MATEUSZ NiKODEM}

Abstract. Given a convex subset $D$ of a vector space and a constant $t \in(0,1)$, a function $f$ : $D \rightarrow \mathbb{R}$ is called $t$-quasiconvex if, for all $x, y \in D$,

$$
f(t x+(1-t) y) \leqslant \max \{f(x), f(y)\} ;
$$

$f$ is called strictly $t$-quasiconvex if, for all $x, y \in D$ such that $f(x) \neq f(y)$,

$$
f(t x+(1-t) y)<\max \{f(x), f(y)\} .
$$

The following Kuhn-type theorem is proved: If $f$ is $t$-quasiconvex and strictly $t$-quasiconvex then it is $(1 / 2)$-quasiconvex and strictly $(1 / 2)$-quasiconvex. It is also shown that lower semicontinuous strictly $t$-quasiconvex functions are quasiconvex, which generalizes the well-known Karamardian's theorem. Mathematics subject classification (2000): Primary 26A51, 39B62.

Keywords and phrases: (Strictly) Quasiconvex functions, (strictly) $t$-quasiconvex functions, Kuhn's theorem, Karamardian's theorem.

\section{REFERENCES}

[1] J. AcZÉL, Lectures on Functional Equations and Their Applications, Mathematics in Science and Engineering, vol. 19, Academic Press, New York-London, 1966.

[2] A. Aleman, On some generalizations of convex sets and convex functions, Anal. Numér. Théor. Approx., 14, 1 (1985), 1-6.

[3] F. BEHRINGER, On Karamardian's theorem about lower semicontinuous strictly quasiconvex functions, Zeitschr. Oper. Res., 23 (1979), 17-48.

[4] F. BEhringer, Convexity is equivalent to midpoint convexity combined with strict quasiconvexity, Optimization, 24 (1992), 219-228.

[5] Z. DARócZY AND Zs. PÁLes, Convexity with given infinite weight sequences, Stochastica, 11, 1 (1987), 5-12.

[6] E. DEÁK, Über konvexe und interne Funktionen, sowie eine gemeinsame Verallgemeinerung von beiden, Ann. Univ. Sci. Budapest. Eötvös Sect. Math., 5 (1962), 109-154.

[7] A. GilÁnyi, K. NiKodem, AND Zs. PÁLES, Bernstein-Doetsch type results for quasiconvex functions, Math. Inequal. Appl., 7, 2 (2004), 169-175.

[8] J. GReenberg AND W. P. Pierskala, A review of quasi-convex functions, J. Operations Res., 19 (1971), 1553-1570.

[9] S. KARAMARDiAn, Srictly quasiconvex (concave) functions and duality in mathematical programming, J. Math. Anal. Appl., 20 (1967), 344-358.

[10] M. Kuczma, An Introduction to the Theory of Functional Equations and Inequalities. Cauchy's Equation and Jensen's Inequality, PWN - Uniwersytet Śląski, Warszawa-Kraków-Katowice, 1985.

[11] N. KuHn, A note on $t$-convex functions, General Inequalities, 4 (Oberwolfach, 1983) (W. Walter, ed.), International Series of Numerical Mathematics, vol. 71, Birkhäuser, Basel, 1984, pp. 269-276.

[12] K. Nikodem And Zs. PÁles, Note on t-quasiaffine functions, Annales Univ. Sci. Budapest., Sect. Comp., 29 (2008), 127-139.

[13] A. W. Roberts And D. E. Varberg, Convex Functions, Academic Press, New York-San Francisco-London, 1973. 